\title{
Investigation of a Flat Connector Concept Potential for Low and Medium Vacuum Conditions
}

\author{
A.B.J.M. Driessen ${ }^{1}$, J. van Duivenbode ${ }^{1,2}$, P.A.A.F. Wouters ${ }^{1}$ \\ 1) Eindhoven University of Technology, the Netherlands \\ 2) ASML, Veldhoven, the Netherlands
}

\begin{abstract}
Higher actuator voltages are required for future generation lithography machines to increase productivity. The operating pressures in combination with relevant insulation distances, can be situated near gas breakdown and cause partial discharge activity, making long-term damage conceivable. High voltage cone-shape connectors with pliable insulation can be applied for the low and medium vacuum range. However, materials and space are restricted making a cone shaped connector not ideal. In this paper, a flat connector concept is presented with a pliable insulation material allowed to be used for the aforementioned application. The permittivity of this material is measured and simulation results of the connector reveal critical design points. A prototype connector is made and its partial discharge inception voltage is characterized for the low and medium vacuum range. The results are discussed and compared with simulations.
\end{abstract}

\section{Introduction}

Future generation lithography machines require a significant increase in actuation power of the moving stages, in order to ensure the continuous demand for improved productivity. Higher current levels to the actuators could achieve this, but such approach requires thicker conductors which increases mass and hampers flexibility. Operation of modern extreme ultraviolet (EUV) lithography machines demand a high flexibility of the cables to ensure a lifespan of a billion movements. The option pursued here, therefore, is higher voltage, i.e. a value possibly exceeding the Paschen minimum, whereas the operating pressure may go down to $0.1 \mathrm{~Pa}$.

The operating range of the EUV machines is in low and medium vacuum, which is generally defined as $10^{2}$ $10^{5} \mathrm{~Pa}$ and $10^{-1}-10^{2} \mathrm{~Pa}$, respectively [1-2]. The first range is used during servicing, the latter range results from the background gas needed for preventing contamination of the optical instruments. Relevant distances in the geometry in combination with these pressure ranges can be situated near the minimum breakdown voltage of the Paschen curve. Long-term damage as a result of partial discharges (PD) is conceivable, when the minimum breakdown voltage for gasses is only a few hundred volts [3-4].
High voltage connectors for low and medium vacuum conditions are not commercially available. Until now, applications have been limited to aerospace. When voltages are limited to approximately $300 \mathrm{~V}$, void containing connectors with sliding contact can be used without expecting lifetime issues [5-6]. This is because the voltage over the voids are below Paschen minimum breakdown voltage [3-4] and no PDs are expected [7]. In cases when the operation voltage exceeds the Paschen minimum, voids can become a potential threat.

A cone shaped connector with soft pliable insulation (e.g. silicone) to eliminate the gas voids between the conductors, offers a reliable connection for hundreds of kilovolts [5-6]. However, the intended application does not require such high voltage levels. On the other hand, materials (e.g. silicone) and space are restricted. A cone shaped connector is therefore not ideal, but based on the same ideology, flat connectors with in between a pliable insulation could offer better prospects. Voids can be allowed to overcome mechanical tolerances, as long as they are located in low electric field regions.

In this paper, a flat prototype connector is presented in Section 2 and its restrictions are investigated in Section 3. Firstly, the permittivity of the soft pliable insulation material (Viton brown for semicon industry), which complies with the material requirements, is measured in order to perform electrostatic voltage simulations. Next, the partial discharge inception voltage (PDIV) of the prototype connector is measured in argon and nitrogen gas. These results are compared with 'scaled Paschen curves' [7] based on electrostatic voltage simulations. Finally, it is investigated whether prior PDs, influence the subsequent PDIV of the connector.

\section{Concept}

A connector provides the ability to split a cable, intended to convey information or energy, from source to load. This division occurs in two consecutive stages, namely the transition from cable to connector or vice versa, and the interface transition of two mating connectors. The two stages of the concept connector are discussed separately.

\subsection{Cable-connector transition}

The goal for the transition from cable to connector is to minimize the electric fields at the cable end in such a 
way that PDs do not occur at maximum operation voltage. A coaxial cable structure for the intended vacuum conditions is necessary to obtain a well-defined ground geometry in order to keep void voltages below the PDIV [7]. This implies that the connector has to continue the coaxial structure and minimize void sizes. Given that the cable-connector transition is a passive mechanical component with no interface, insulating potting material is an easy option to accomplish this transition, making this a relatively easy transition. For the prototype connector, a low viscosity epoxy with minimum outgassing was chosen to meet cleanroom standards. In addition, geometric field control measures were established with the components 'E-field control threaded bushing', 'core bushing' and 'shield bushing' shown in Fig. 1a.

\subsection{Interface transition}

The transition between two mating connectors is more challenging. The use of an insulating potting material is not possible for the interface as this would result in a permanent joint. Connectors with sliding contacts have voids as a result of mechanical tolerances. These voids, especially in radial direction for coaxial structures, are susceptible for PDs when their voltage differences exceed the Paschen breakdown voltage for the respective pressure [7]. A solution is to allow having voids in low E-field regions or to reduce the void size with soft pliable insulation material. An example that uses both techniques, is the cone shaped connector which is able to handle hundreds of kilovolts [5-6]. Disadvantage of the cone shaped connector is its dimensions; they become too large for the intended application. Voltage levels for the intended application, though being well above the Paschen minimum, remain limited to a few kilovolts. The proposal of this paper is therefore to use a flat connector interface with a coaxial structure along with a flat O-ring shaped pliable insulation material, see Fig. 1a. Core and shield voids are allowed, since their local E-field strength is reduced by adding the 'core bushing' and 'shield bushing' at each connector. Both connectors are female type (Fig. 1b-1d) and equipped with a 'heavy mate female single contact'. Together with a separate 'connection pin' (Fig. 1c), a conductive path for the core conductor is established. The 'connection nut' realizes a similar situation (Fig. 1a-1b), but for the shield conductor. It is provided with venting holes to equalize the pressure of the 'shield void' with the environment. The insulation material at the connector interface is the same used for the cable-connector transition. The flat interface requires adequate polishing to minimize voids size. The connectors are provided with semi-rigid coaxial cable for testing purposes.

\section{Results \& Discussion}

In this section, the prototype connector is tested. Its performance and limitations are discussed in three topics:

- The permittivity of the soft pliable insulation material is determined together with static voltage and E-field simulations of the connector.

- The PDIV of the realized connector is measured as function of pressure and compared with prior simulation results. This is possible by scaling the Paschen curves for homogeneous fields to take into account the different path lengths and associated voltage drops. For convenience, we shall refer to them as scaled Paschen curves (s.P.c).

- Possibly charge accumulates on the soft pliable insulation material. The influence of prior PDs on the PDIV of the connector is investigated.

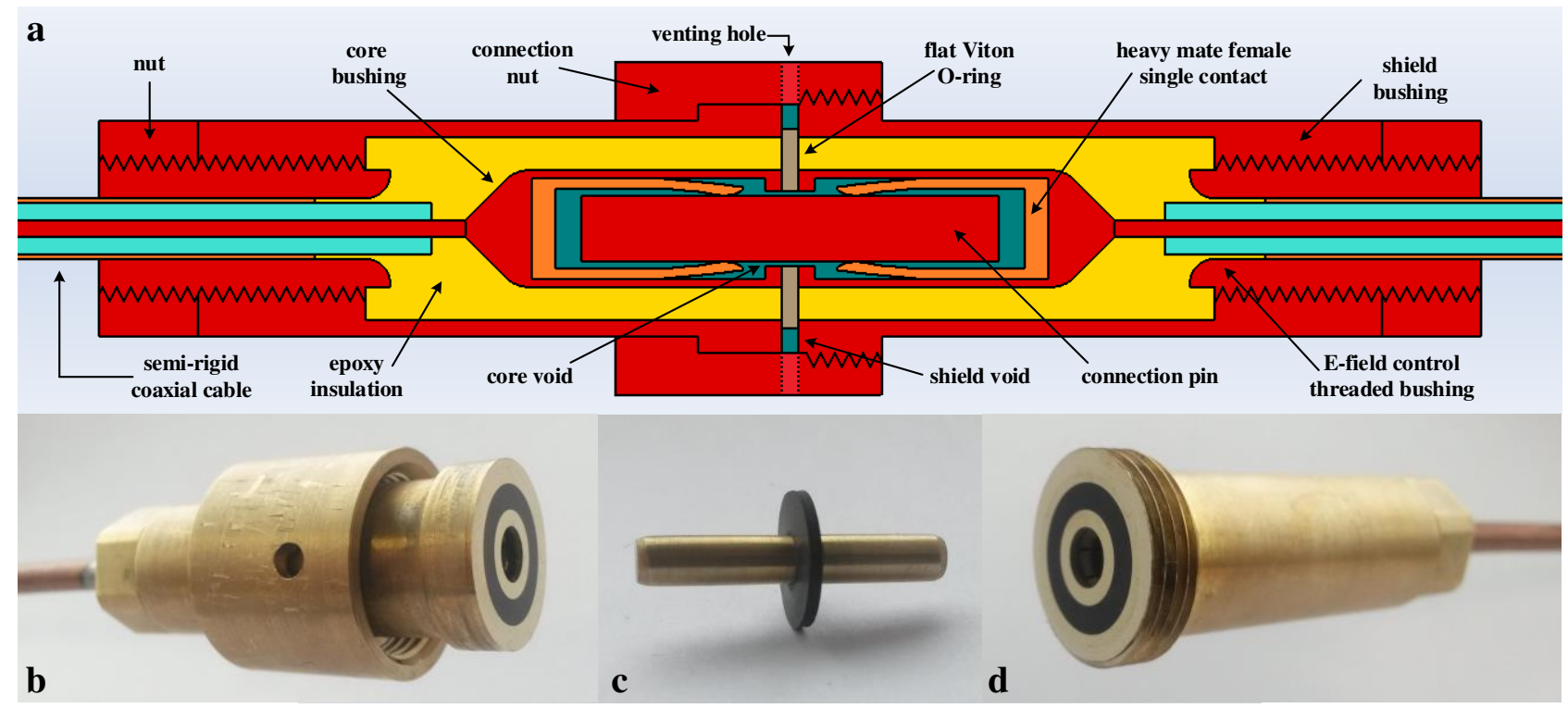

Fig. 1 - Two jointed prototype coaxial connectors equipped with semi-rigid coaxial cables, a: schematic view in axial direction provided with a description of the main components, b: realization of left connector with connection nut, c: realization of connection pin with flat shaped Viton brown O-ring, d: realization of right connector. 


\subsection{Permittivity insulation material}

For the interface of the prototype connectors, a soft pliable insulation material is required to minimize void size. For the application, materials are restricted to prevent contamination of the EUV optics. Silicone may therefore not be used, but a certain type of Viton brown, specially designed for the semicon industry, is allowed. Unfortunately, the permittivity is unknown and has to be determined to perform electrostatic voltage simulations.

To this end, a disc capacitor is made with brass electrodes glued with a thin layer of epoxy to the soft pliable insulation material, see Fig. 2. The capacitance $\mathrm{C}_{\text {meas }}$ is measured for different frequencies with two different LCR meters (Agilent 4263B, Hameg 8118), and the relative permittivity $\varepsilon_{\mathrm{r}}$ is calculated according to [8] with:

$$
\begin{gathered}
\epsilon_{r}=\frac{C_{\text {meas }}-C_{e}}{C_{n}} \\
C_{e}=1 / 2 \varepsilon_{0} D\left[\left(\ln \frac{8 \pi D}{b}\right)-3+\right. \\
\left.\left(1+\frac{t}{b}\right) \ln \left(1+\frac{t}{b}\right)-\frac{t}{b} \ln \frac{t}{b}\right] \\
C_{n}=\frac{\epsilon_{0} \pi D^{2}}{4 b}
\end{gathered}
$$

were $\mathrm{D}$ and $\mathrm{t}$ are the electrode diameter and thickness respectively, and $\mathrm{b}$ the distance between the inner faces of the electrodes. The relative permittivity shows linear behavior over the measured frequency domain (Fig. 3), and has an extrapolated value of 13.2 for $50 \mathrm{~Hz}$.

The capacitance of the disc capacitor is simulated with a 2D rotational symmetric solver (Electro from Integrated Engineering Software) for different values of the relative permittivity, see Fig. 4 . In addition, the measured results with corresponding permittivity are included in this figure. The measurements and simulations are in good agreement and show linear behavior.

Figure 5 shows the static voltage distribution simulation result of two joint connectors with $1 \mathrm{kV}_{\mathrm{DC}}$ applied on the core and a grounded shield. A zoomed view of the shield void is shown in the inset of Fig. 5, which indicates that the maximum void voltage difference is approximately $36 \mathrm{~V}_{\mathrm{DC}}$ for a flat Viton brown O-ring with an outer diameter of $12.0 \mathrm{~mm}$.

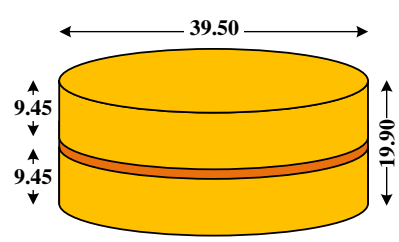

Fig. 2 - Schematic view of the disc capacitor in millimeters with Viton brown as dielectric material and brass electrodes.

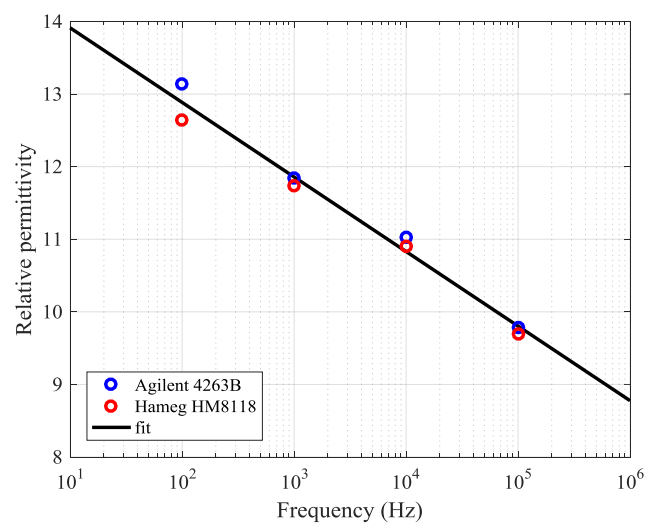

Fig. 3 - Dielectric constant as function of frequency of the Viton brown disc capacitor.

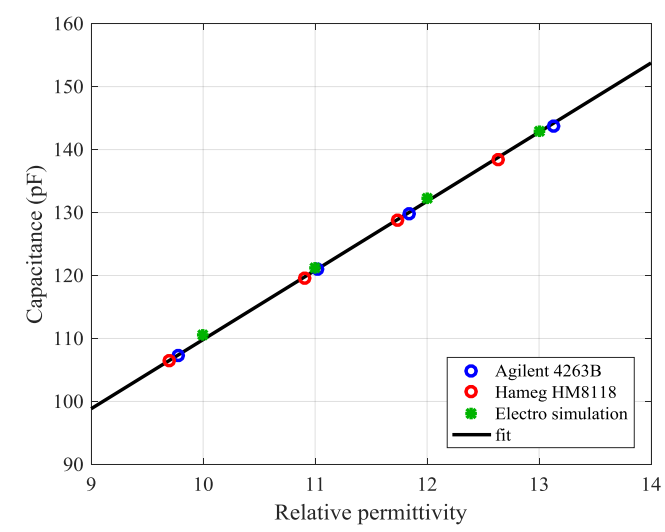

Fig. 4 - Capacitance of Viton brown disc capacitor as function of dielectric constant.

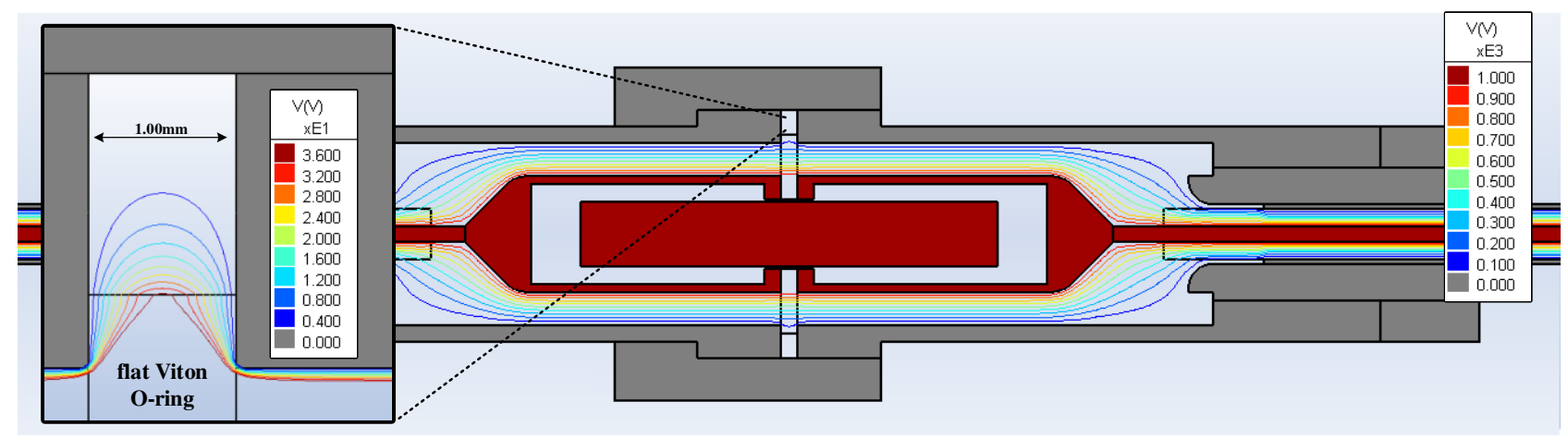

Fig. 5 - Static voltage distribution simulations of two joint connectors viewed in axial direct with a close-up taken at the shield void. 


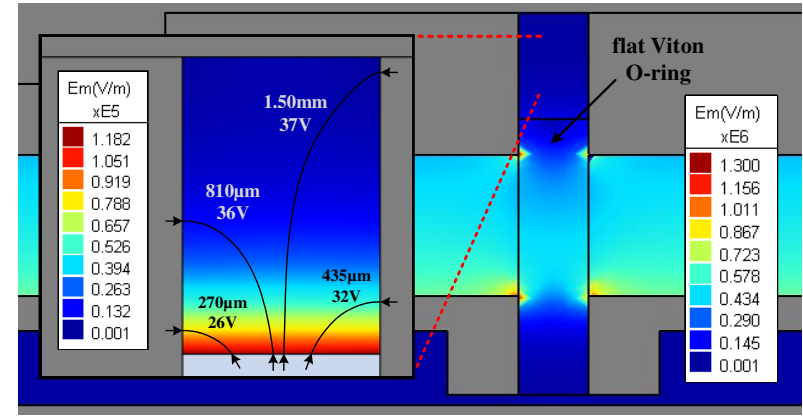

Fig. 6 - Static electric field simulations near the connection interface of two connectors with a close-up at the shield void.

The cable-connector transition shows smooth equipotential lines. For the interface transition near the voids, however, a static electric field simulation (Fig. 6) reveals field enhancement at the material transition of brass, epoxy and Viton brown. This is caused by differences in material permittivity and geometry, but also due to limitations of the simulation technique. Perfect rectangular shapes induce significant simulation errors near the edges, but can be solved by introducing a rounding radius. However, due to the polishing process of the connector interface, it is very hard to define a radius and obtain reproducible results. This could probably be a critical point in the design of the flat connector and requires further research. For now, the prototype connector of Fig. 1 will be tested, and if this is currently an obstacle, this will be revealed during PDIV characterization.

\subsection{Partial discharge inception voltage}

Characterization of the PDIV reveals the maximum operation levels in order to guarantee long-term operation without damage or failure. A schematic overview of the PDIV characterization setup, consisting of the connector under test, electrical system and vacuum system, is depicted in Fig. 7. Detailed information about installation and operation is reported in [7]. Applied voltage and gas pressure in the vessel are computer controlled. The gas, either argon or nitrogen, has a purity of N5.0. The connector under test is placed in a vessel and is connected via semi-rigid coaxial cables and terminations to PD detection equipment. These cables do not produce PDs during the characterization of the connector, because they are lacking voids. The venting hole in the 'connection nut' ensures that pressures are equal between vacuum vessel and 'shield void'. The narrower voids along the core remain at atmospheric pressure and do not cause discharge activity.

The test procedure involves measurement of the PDIV over a wide range of pressures. During each measurement, the pressure in the vacuum vessel is monitored to obtain the mean value and standard deviation. In this paper, the PDIV is defined as the voltage from which PDs occur with a minimum apparent charge of $100 \mathrm{fC}$ for at least $10 \mathrm{~s}$. This level is chosen since it clearly above the background noise level (typically $40 \mathrm{fC}$ ). The PDIV is found by increasing the voltage, starting with a 'fast' rise time (approximately $67 \mathrm{~V}_{\mathrm{rms}}$ every $2 \mathrm{~s}$ ), followed by a 'slow' increase (approximately $1.7 \mathrm{~V}_{\mathrm{rms}}$ every $4 \mathrm{~s}$ ). An estimate of the PDIV is based on prior measured results and when reaching $1 \mathrm{kVrms}$ below the expected PDIV value, the rate of increase is lowered.

In total 40 and 22 measurements are taken for argon and nitrogen gas respectively. Pressure deviations are kept within $2.3 \%$ of the nominal pressure. The measurement results for argon are displayed in Fig. 8. The cable terminations are located in an air environment at ambient pressure, see Fig. 1, and the connector is in the vacuum vessel at the selected pressure. The results show two distinct patterns. The left part with the PDIV nearly constant having a value just above $5 \mathrm{kV}_{\text {rms }}$ is related to the PD inception occurring in the terminations located outside the vessel. This was confirmed by adding a small amount of $\mathrm{SF}_{6}$ gas nearby the termination, resulting in an increase of the PDIV [7]. The right part with a convex relation is related to PDs occurring inside the 'shield void' and can be associated with the Paschen law as discussed hereafter. The minimum PDIV is $2.5 \mathrm{kV}_{\text {rms }}$ at $3.05 \mathrm{kPa}$. The measurements for nitrogen are all nearly constant and have a value just above $5 \mathrm{kV}_{\text {rms. }}$ The PDs originate from the cable terminations. The current configuration does not allow us to characterize the connector for a nitrogen environment.

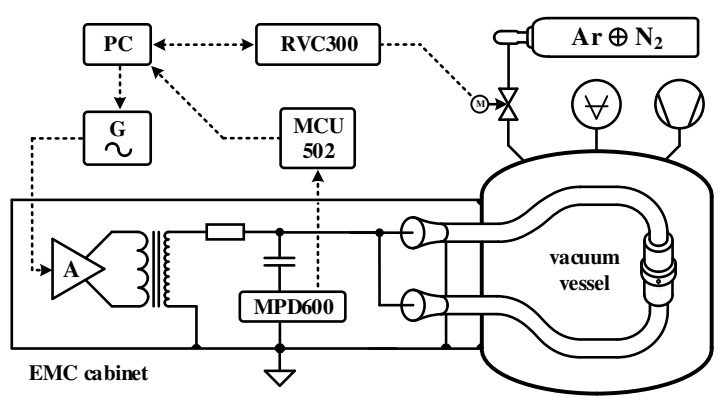

Fig. 7 - Experimental setup consisting of the connector under test, electrical system and vacuum system.

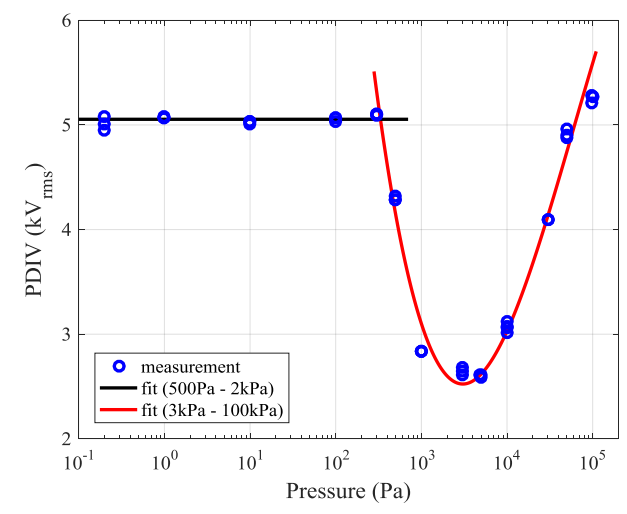

Fig. 8 - Partial discharge inception voltage as a function of gas pressure for the connector in argon. 


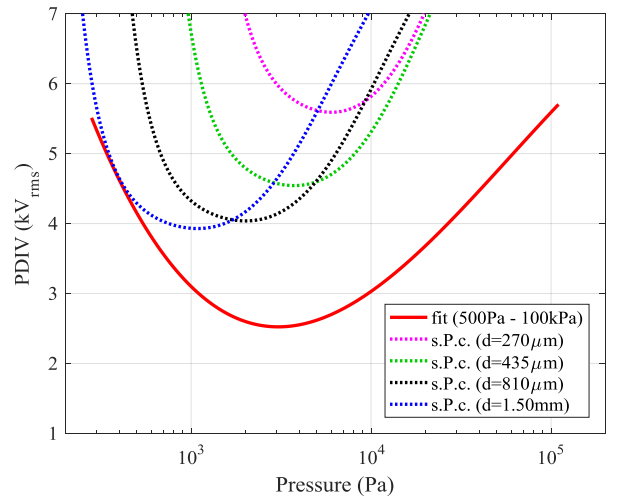

Fig. 9 - Scaled Paschen curves of the connector void together with a fit of the PDIV of the connector in argon gas.

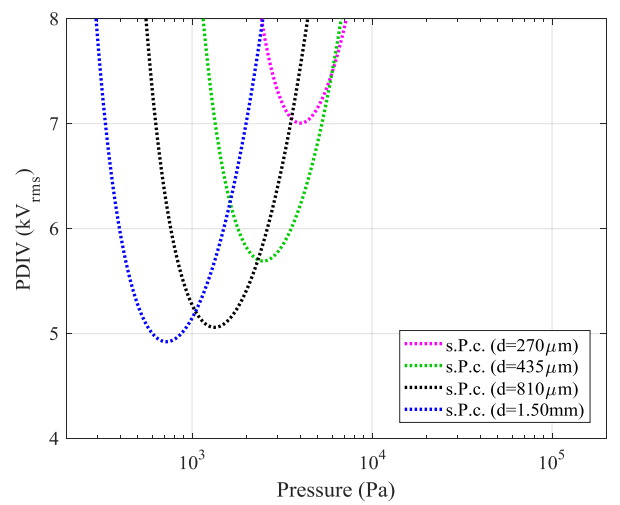

Fig. 10 - Scaled Paschen curves of the connector void in nitrogen gas.

When applying a voltage on the core conductor with respect to the shield conductor at the connector interface, part of the voltage drops over the soft pliable insulation and only a fraction over the shield and core voids. The voltage over the first dominant gap together with the pressure and gas type determines the PDIV according to the Paschen law. To account for this fraction, scaling is applied. By means of electrostatic field calculations (Fig. 5), the fraction of the applied voltage over a void is determined. A scaling factor $\alpha$ can be defined relating the void voltage $V_{\text {void }}$ along a discharge path to the Paschen breakdown voltage $V_{b r}$ along this path:

$$
\alpha=\frac{1}{\sqrt{2} V_{\text {void }}} V_{b r}
$$

This factor must be multiplied with the simulation voltage $\left(1 \mathrm{kV}_{\mathrm{DC}}\right)$ to obtain the voltage to be applied on the conductor to reach the PDIV. Note that the PDIV is expressed as rms voltage. The breakdown voltages $\mathrm{V}_{\mathrm{br}}$ for argon and nitrogen are taken from the Paschen curves provided in [3] and [4] having a minimum value of $206 \mathrm{~V}_{\mathrm{DC}}$ and $258 \mathrm{~V}_{\mathrm{DC}}$, respectively. Inside the connector interface different trajectories, along which discharges can develop, are simultaneously present. The PDIV is determined by the trajectory which results in the lowest $\mathrm{V}_{\mathrm{br}}$. This depends on the voltage fraction over this distance in relation to the product pressure and distance according to the Paschen curves.

The inset of Fig. 6 show a few discharge trajectories. For these trajectories the s.P.c.'s are plotted in Fig. 9 for argon and Fig. 10 for nitrogen. The maximum voltage inside the void arises halfway on top of the Viton Oring. From this region the longest discharge paths arise. Left and right of this region the voltage is less, resulting in higher conductor voltages needed to reach inception along the corresponding paths. However, the discharge distances are shorter, resulting in Paschen curves being shifted upwards and to the right.

In Fig. 9 the continuous curve represents the fit based on the experimental data for argon from Fig. 8. It is clearly observed that the PDIV measured is significantly lower than expected from simulations. The measured lowest value is $2.5 \mathrm{kV}_{\text {rms }}$ whereas the model predicts a voltage of $3.9 \mathrm{kV}_{\text {rms }}$, a deviation factor of 1.56. For nitrogen the PDIV for the connector design was not reached at conductor voltages up to the level that the terminations start discharging. The curves in Fig. 10 indeed are close to this voltage level for any pressure value.

The hypothesis for the deviation is that due to discharges, charge accumulates on the Viton material, resulting in increased electric field strength when the voltage is reversed. This causes a reduction of the applied voltage level to reach inception of PDs in the void. For nitrogen the PDIV is not reached at all, so no charge has accumulated from prior PDs.

\subsection{Charge accumulation}

Surface potential as a result of charge accumulation on insulation materials is a common phenomenon. The surface potential decay of polymeric materials is investigated for different materials [9-11], but no information is available on charge accumulation for this particular insulation material, nor for similar fluorelastomers. To determine whether charge can accumulate as a result of prior PDs, the PDIV of the connector is measured multiple times consecutively at a fixed pressure. However, prior to the first measurement, an idle period is introduced allowing surface charge to leak away. The required duration is not known beforehand, but a minimum of over 10 hours was taken as an educated guess [9-11].

The consecutive PDIV measuring results in argon for different pressures are displayed in Fig. 11. In total 17 measurements are performed, five at $1.5 \mathrm{kPa}$ and $3.0 \mathrm{kPa}$, and seven for $1.0 \mathrm{kPa}$. The idle period prior to the first measurement is $>10^{4}, 20$ and 14 hours for increasing pressure, respectively. The first measurement at $1.5 \mathrm{kPa}$ and $3.0 \mathrm{kPa}$, and the first two at $1.0 \mathrm{kPa}$ are significantly higher compared to the values in the subsequent measurements.

The results of the consecutive PDIV measurements infer that surface potential can arise on insulation material as 
a result of PDs. From this experiment, it is however unknown how the surface potential behaves as a result of applied charge and time. A striking observation is that the PDIV ratio of the first measurement and the mean of the subsequent measurements at $3.0 \mathrm{kPa}$, the pressure at which the lowest PDIV is observed. The ratio of 1.67 is close to the ratio of the simulated and measured PDIV in Section 3.2.

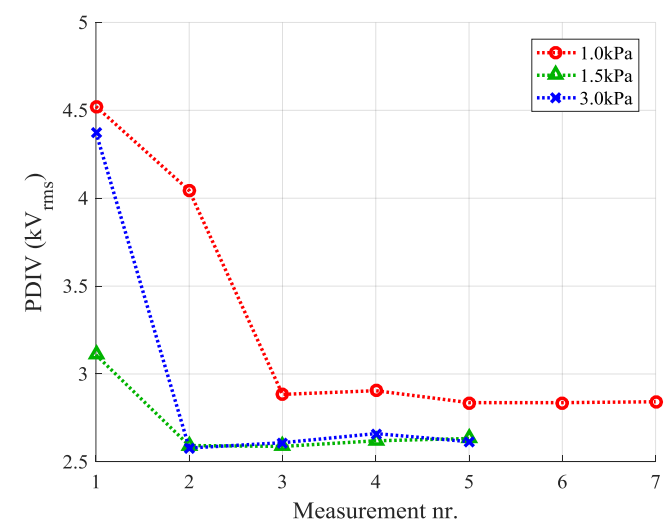

Fig. 11 - Partial discharge inception voltage of consecutive measurements at different pressures in argon. The idle period prior to the first measurement is $>10^{4}$ hours $(1.0 \mathrm{kPa}), 20$ hours $(1.5 \mathrm{kPa})$ and 14 hours $(3.0 \mathrm{kPa})$.

\section{Conclusions}

The proposed flat connector has good potential for applications with high voltage, limited to few kilovolts, in low and medium vacuum conditions. The tested prototype connector has minimum PDIV of $2.5 \mathrm{kV}_{\mathrm{rms}}$ in argon as a result charge accumulation on the insulation surface due prior PDs. According to electrostatic voltage simulations, the expected minimum PDIV is $4.0 \mathrm{kV}_{\mathrm{rms}}$ when no charge is present on the insulation. This would be more than sufficient for application in lithographic machines which at present aim for $2 \mathrm{kV}_{\mathrm{pk}-\mathrm{pk}}$.

The permittivity of the pliable insulation material is measured and the simulation results of the prototype connector reveals electric field enhancement near the material transition of brass, epoxy and Viton brown. This is caused by differences in material permittivity and geometry, but also limitations of the simulation technique. This could probably be a critical point in the design of the flat connector and requires further research. The tested prototype connector does not show signs of damage due to electric field stress. The effect of charge accumulation and its decay should still be studied in more detail.

The intention of the flat connector concept is to mimic the insulation concept of the cone-shaped connector but decrease volume at the expense of maximum voltage levels. The tested prototype connector has proven that the insulation concept works, but there is still room for improvement in terms of volume reduction. The electrical connection for the core conductors of the connectors is a dominant factor in the design due to the size of 'heavy mate female single contact' in Fig. 1a. A different spring mechanism for the electrical connection, e.g. located in the 'connection nut', can offer an improved usage of available space.

\section{References}

[1] Oerlikon Leybold Vacuum GmbH, Fundamentals of Vacuum Technology, Cologne, Germany, Jun. 2007.

[2] Pfeiffer Vacuum GmbH, Vacuum Technology Know How, Asslar, Germany, Mar. 2009.

[3] M. Klas, Š. Matejčík, B. Radjenović, M. Radmilović-Radjenović, "Experimental and theoretical studies of the direct-current breakdown voltage in argon at micrometer separations", in Physica Scripta, Vol. 83, No. 4, Mar. 2011.

[4] T. W. Dakin et al., "Breakdown of gases in uniform fields - Paschen curves for nitrogen, air and sulfur hexafluoride", Electra (CIGRE, Paris), No. 32, pp. 6182 (Jan. 1974).

[5] W. G. Dunbar, "High voltage design guide: aircraft", Boeing Aerospace Co., Seattle, WA., Vol. IV, Jan. 1983.

[6] W. G. Dunbar, W. J. Seabrook, "High voltage design guide for airborne equipment", Boeing Aerospace Co., Seattle, WA., Jun. 1976.

[7] A. B. J. M. Driessen, J. van Duivenbode and P. A. A. F. Wouters, "Non-hermetic cable connection to enable high voltage in low \& medium vacuum", 2016 IEEE Conference on Electrical Insulation and Dielectric Phenomena (CEIDP), Toronto, ON, 2016, pp. 424-427.

[8] A. H. Scott, H.L. Curtis, "Edge correction in the determination of dielectric constant", Journal of Research of the National Bureau of Standards, Vol. 22, Jun. 1939.

[9] Y. Zhuang, G. Chen, P. H. Chappell and M. Rotaru, "Surface potential decay: Effect of different corona charging times", 2012 Annual Report Conference on Electrical Insulation and Dielectric Phenomena, Montreal, QC, 2012, pp. 620-623.

[10] G. Chen, “A new model for surface potential decay of corona-charged polymers", Journal of Physics D: Applied Physics, Vol. 43, No. 5, Jan. 2010.

[11] S. Kumara, B. Ma, Y. V. Serdyuk and S. M. Gubanski, "Surface charge decay on HTV silicone rubber: effect of material treatment by corona discharges", IEEE Transactions on Dielectrics and Electrical Insulation, Vol. 19, No. 6, Dec. 2012. 